\title{
Una nueva especie de Hyphessobrycon (Characiformes: Characidae) de la cuenca del río Telembí, vertiente sur del Pacífico, Colombia
}

\author{
Carlos A. García-Alzate ${ }^{1}$, César Román-Valencia ${ }^{2} \&$ Donald C. Taphorn ${ }^{3}$ \\ 1. Departamento de Biología, Universidad del Atlántico, km 7 antigua vía a Puerto Colombia, Barranquilla, Atlántico \\ Colombia; carlosalzate@mail.uniatlantico.edu.co \\ 2. Universidad del Quindío, Laboratorio de Ictiología, A. A. 2639, Armenia, Quindío, Colombia; \\ ceroman@uniquindio.edu.co \\ 3. 1822 North Charles Street, Belleville, Illinois, 62221, USA; taphorn@gmail.com
}

Recibido 07-XI-2011. Corregido 24-VI-2012. Aceptado 26-VII-2012.

\begin{abstract}
A new species of Hyphessobrycon (Characiformes: Characidae) from the Telembí River drainage, Southern Pacific slope of Colombia. The genus Hyphessobrycon is included within the subfamily Tetragonopterinae. The species are generally small, do not exceed $70 \mathrm{~mm}$ of standard length, are economically important as ornamental fish, with 128 valid species distributed from Southern Mexico to Rio La Plata in Argentina. The collections of fish were made with seines, in a single biotope, along shore in backwaters and working downstream. Measurements of the specimens were taken point to point with digital calipers. Observations of bone and cartilage structures were made on cleared and stained (C\&S) specimens. The morphometric relationships between species were explored using a principal component analysis (PCA)using 21 variables. We described a new species, Hyphessobrycon chocoensis, from the Telembí River drainage of the Pacific versant of Colombia. The new species, Hyphessobrycon chocoensis, is distinguished from congeners not of the "flammeus" species group by: having a diffuse humeral spot, in lacking a dark spot on the dorsal fin and caudal peduncle. It is distinguished from members its species group by the number of rays in the dorsal fin (ii, 8 , i), by the number of branched anal-fin rays (25-26) and by having a diffuse humeral spot. It differs from $H$. tortuguerae in the number of teeth on the maxilla (1-2), by the number of predorsal scales and the high number of scales between the lateral line and the anal fin (6-7). Hyphessobrycon chocoensis can be distinguished from the other known species of Hyphessobrycon from the Pacific Coast of Colombia in having: a high number of pored lateral-line scales, by the snout to dorsal-fin length, by caudal-peduncle depth and by eye diameter. In addition, it differs from $H$. columbianus by the distance from the dorsal fin to the anal fin, by the length of the upper jaw, and by snout length. It differs from $H$. condotensis in having a high number of scales between the lateral line and the dorsal fin, and by the number of simple rays in the anal fin. H. chocoensis is distinguished from $H$. sebastiani by the number of transverse scales and the number of scales between the lateral line and the dorsal fin. A taxonomic key is provided to aid with the identification of the species of Hyphessobrycon from the Pacific Coast of Colombia. Rev. Biol. Trop. 61 (1): 181-192. Epub 2013 March 01.
\end{abstract}

Key words: new taxon, new species, tropical fish, Choco biogeographic area, tetra.

El género Hyphessobrycon, se incluye dentro de la subfamilia Tetragonopterinae (sensu Mirande, 2010). Las especies generalmente son pequeñas, no sobrepasan los $70 \mathrm{~mm}$ de longitud estándar, muchas son de importancia económica como peces ornamentales, y actualmente están distribuidos en todas las grandes cuencas de la región neotropical, desde el sur de México hasta el río de la Plata en Argentina.
Aunque 157 especies han sido ubicados en Hyphessobrycon, debido al reconocimiento de sinónimos o la reubicación de algunas especies en otros géneros, hoy se consideran válidas 128 especies (Eschmeyer \& Fricke 2011). De estas, 21 especies de Hyphessobrycon han sido identificadas para Colombia (García-Alzate et al. 2010c): H. acaciae García-Alzate, RománValencia \& Prada-Pedreros, H. amaronensis 
García-Alzate, Román-Valencia \& Taphorn, H. bentosi Durbin, H. columbianus Zarske \& Géry, H. condotensis Regan, H. diancistrus Weitzman, H. ecuadoriensis Eigenmann \& Henn, H. erythrostigma Fowler, H. heterorhabdus (Ulrey), H. mavro García-Alzate, Román-Valencia \& Prada-Pedreros, H. metae Eigenmann \& Henn, H. niger García-Alzate, Román-Valencia \& Prada-Pedreros, H. ocasoensis García-Alzate \& Román-Valencia, $H$. oritoensis García-Alzate, Román-Valencia \& Taphorn, H. panamensis Durbin, H. poecilioides Eigenmann, $H$. proteus Eigenmann, $H$. sweglesi Géry, H. sebastiani García-Alzate, Román-Valencia \& Taphorn, H. saizi Géry, H. taguae García-Alzate, Román-Valencia \& Taphorn, de las cuales tres se distribuyen en el Chocó biogeográfico, vertiente Pacífico Colombiano y pertenecen al grupo flammeus: H. columbianus, $H$. condotensis y H. sebastiani.

En Hyphessobrycon aun no existe un consenso para la definición de clados filogenéticos, sin embargo, se han reconocido seis grupos convenientes, pero artificiales, de especies basados en modelos de pigmentación (Géry 1977, Weitzman \& Palmer, 1997). Estos son: (a) especies que carecen completamente de marcas negras en el cuerpo (H. panamensis); (b) especies con una o dos manchas humerales (H. flammeus); (c) especies con solo una mancha en el pedúnculo caudal (H. minimus); (d) especies con una mancha humeral y peduncular (H. tropis); (e) especies con un patrón longitudinal, consistente en una franja horizontal lateral que se extiende desde la región humeral hasta la caudal o, a veces, una franja interrumpida con la mancha humeral aun visible $(H$. heterorhabdus); y (f) especies con una mancha oscura en la aleta dorsal ( $H$. callistus). Todos los grupos de especies tienen representantes en Colombia. Actualmente García-Alzate \& Román-Valencia (2008) y García-Alzate et al. (2008a, b, c, 2010 a, b, c, 2011) estudian la taxonomía alfa de las especies del grupo heterorhabdus presentes en Colombia y Venezuela. El objetivo del presente trabajo fue describir una nueva especie de Hyphessobrycon del grupo flammeus encontrada en la cuenca del río
Telembí en la vertiente del Pacifico de Colombia. Se incluye una clave taxonómica de las especies trans-andinas de Colombia.

\section{MATERIALES Y MÉTODOS}

Las recolectas de los peces se realizaron con arrastres con una malla fina (ojo de malla 6.0 por $3.5 \mathrm{~mm}$ ) de $3.85 \mathrm{~m}$ de longitud por $1.65 \mathrm{~m}$ de ancho. Las capturas se hicieron en un solo biotopo zona litoral de remanso y a favor de la corriente. Los ejemplares se depositaron en la colección de peces del museo de la Universidad de Auburn, Alabama, USA (AUM) y en la colección de peces del laboratorio de Ictiología del programa de Biología, Universidad del Quindío, Armenia, Colombia (IUQ). Las medidas de los ejemplares se tomaron punto a punto con un calibrador digital, los datos del holotipo se indican con asterisco $(*)$. Las medidas y conteos (Cuadro 1) se realizaron, siempre que fue posible, sobre el lado izquierdo de los ejemplares. Medidas y conteos siguen a Fink \& Weitzman (1974) y se expresaron como porcentaje de la longitud estándar (LE) y porcentaje de la longitud cabeza (LC). Las medidas del material tipo de Hyphessobrycon condotensis y H. ecuadoriensis (grupo flammeus) fueron tomadas a partir de fotos digitales, con el programa Scion Image, bajo Windows versión 4.0.3.2. Las relaciones morfométricas entre especies fueron exploradas empleando 21 variables (Cuadro 1). Se realizó un análisis de componentes principales (ACP), se utilizó el método Burnaby para eliminar la influencia de la talla sobre la forma, por medio del programa PAST versión 1.81 (Hammer et al. 2008). El número de ejemplares y el rango de medidas mínimas y máximas de cada lote examinado se presentan en paréntesis.

Las observaciones de estructuras óseas y cartílagos se hicieron sobre ejemplares clareados y teñidos (C\&T) de acuerdo a los métodos descritos por Taylor \& Van Dyke (1985) y Song \& Parenti (1995). La nomenclatura de huesos se basó en Weitzman (1962) y Vari (1995). Coordenadas y altitud fueron registradas con un sistema electrónico portátil de posición 
CUADRO 1

Datos morfométricos de Hyphessobrycon chocoensis sp. n.

TABLE 1

Morphometric data Hyphessobrycon chocoensis n. sp.

\begin{tabular}{|c|c|c|c|}
\hline Morfometría & Holotipo & Paratipos & DS \\
\hline Longitud estándar & 37.0 & $25.5-37.0(31.1)$ & 4.2 \\
\hline Longitud total & 48.7 & $33.1-48.7(40.4)$ & 5.3 \\
\hline \multicolumn{4}{|l|}{ Porcentaje de la longitud estándar } \\
\hline 1. Profundidad del cuerpo & 42.6 & $35.8-42.6(39.3)$ & 2.2 \\
\hline 2. Longitud hocico-aleta dorsal & 51.1 & $41.3-52.3(49.9)$ & 3.3 \\
\hline 3. Longitud hocico-aletas pectorales & 27.3 & $26.8-30.0(28.6)$ & 1.3 \\
\hline 4. Longitud hocico-aletas pélvicas & 45.8 & $36.9-46.3(43.7)$ & 2.9 \\
\hline 5. Longitud hocico-aleta anal & 59.3 & $56.5-61.6(59.1)$ & 1.9 \\
\hline 6. Longitud aleta dorsal-hipurales & 51.4 & $48.0-53.5(51.1)$ & 1.8 \\
\hline 7. Longitud aleta dorsal-aleta anal & 38.0 & $32.7-42.9(37.5)$ & 2.8 \\
\hline 8. Longitud aleta dorsal-aletas pectorales & 42.6 & $38.4-46.8(41.6)$ & 2.4 \\
\hline 9. Longitud aleta dorsal & 32.5 & $27.5-34.0(31.3)$ & 2.3 \\
\hline 10. Longitud aletas pectorales & 21.8 & $20.0-23.5(21.4)$ & 1.1 \\
\hline 11. Longitud aletas pélvicas & 17.9 & $17.6-20.0(18.5)$ & 0.9 \\
\hline 12. Longitud aleta anal & 20.7 & $20.5-23.2(21.6)$ & 0.9 \\
\hline 13.Profundidad del pedúnculo caudal & 8.0 & $7.4-10.5(9.0)$ & 1.1 \\
\hline 14. Longitud del pedúnculo caudal & 11.9 & $9.6-12.3(11.1)$ & 0.7 \\
\hline 15. Longitud de la cabeza & 28.1 & $26.6-30.1(28.2)$ & 0.9 \\
\hline \multicolumn{4}{|l|}{ Porcentaje longitud cabeza } \\
\hline 16. Longitud del hocico & 17.3 & $16.8-18.0(17.3)$ & 0.4 \\
\hline 17. Diámetro del ojo & 40.3 & $40.3-49.9(43.9)$ & 2.6 \\
\hline 18. Longitud post-orbital de la cabeza & 31.9 & $29.2-37.8(32.9)$ & 2.5 \\
\hline 19. Longitud del hueso maxilar & 41.9 & $36.3-43.2(40.9)$ & 2.5 \\
\hline 20. Ancho interorbital & 43.1 & $37.9-44.5(41.1)$ & 2.5 \\
\hline 21. Longitud mandíbula superior & 13.7 & $13.7-16.4(15.0)$ & 1.1 \\
\hline
\end{tabular}

*Longitud estándar y total en mm, promedio entre paréntesis. DS=desviación estándar. N=49/Total and standard length in $\mathrm{mm}$, average in parentheses. $\mathrm{SD}=$ standard deviation. $\mathrm{N}=49$.

global Magellan GPS4000XL. En la descripción el número de ejemplares se presenta entre paréntesis. Abreviaciones institucionales (IUQ, AUM y del material examinado) siguen a Sabaj-Pérez (2010).

Material de comparación examinado: Hyphessobrycon condotensis: $\mathrm{BMNH}$ 1913.10.1.19-21, syntypes (3) (25.9-31.2mm de LE), Colombia, Río Condoto and Río San Juan, 1913. ICNMHN 2278, (2) (27.6-34.7mm de LE) Colombia, Vía de Pepé, Baudó Chocó. Hyphessobrycon columbianus: IMCN 242, (5) (29.8-39.8mm de LE) Colombia, Río Guati,
Acandí, Chocó, 1 ago 1995; MCNG 47820, (1), Colombia, Río Guatí, Chocó, 15 ago 1995. Hyphessobrycon compressus: FMNH 4641, holotipo, México, Obispo, Vera Cruz; FMNH 4662, paratipos (1 C\&T) (29.2mm de LE) recolectado con el holotipo; BMNH-1905.12.6.45, paratipos, (2) (30.6-31.6mm de LE) México, Obispo, Vera Cruz; IBUAM-P 8538, (2) (24.5$31.2 \mathrm{~mm}$ de LE) México, Trinitaria, Flor de Café, Chris, 3 jul 1993; ANSP 124774, (12) (30.3-35.6mm de LE) México, Río Usumacinta casi unido con Pasión, cerca Sayache, 18 ago 1961; ANSP 124774, (3 C\&T) (30.6-35.6mm de LE) México, Río Usumacinta casi unido con 
Pasión, cerca Sayache, 18 ago 1961. Hyphessobrycon ecuadoriensis: CAS 61602, holotipo (24.3mm de LE) Ecuador, Los Ríos, cerca a Vinces. BMNH 1924.3.3.44-45, paratipos (2) (22.6-25.5mm de LE), Ecuador, Los Ríos, cerca a Vinces; MEPN 85-117, (4), Ecuador, Brazo del Río Bravo $1 \mathrm{~km}$ aguas arriba del salto del Bravo, Esmeraldas, 11 ago 1985. Hyphessobrycon panamensis: MCZ 20688, syntype (1) $(23.5 \mathrm{~mm}$ de LE) Río Boquerón, vertiente Atlántico, Panamá. AMNH 31802, (13) (27.6$33.4 \mathrm{~mm}$ de LE) Panamá, Zona del canal de Panamá, Quebrada Juan Grande Cerca a Gamboa, 1978; IUQ 601, (1 C\&T) (25.5mm de LE) Colombia, Nariño, Tumaco, El Pinde, afluente río Pianurbi en la vía Llorente-Guayacana, 19 jul 2000; IUQ 2285, (19) $(26.5-34.3 \mathrm{~mm}$ de LE) Costa Rica, Limón, río Cocolis, $35 \mathrm{~km}$ SE de Shiroles, 6 oct 1979. Hyphessobrycon sebastiani: IUQ 1942, holotipo, (39.5 mm SL, male) Colombia, Pacific versant, Chocó, Istmina, San Juan River drainage, Patecucho Creek, approximately $5^{\circ} 09^{\prime} \mathrm{N}-76^{\circ} 40^{\prime} \mathrm{W}, 7$ August 2002, T. Silirio. ver García-Alzate et al. (2010a). Hyphessobrycon savagei: IUQ
2310, (30) (25.5-39.9mm de LE) Costa Rica, Quebrada Puntarenas, 200m SE del Río Nuevo Salama, 5 ene 1967. Hyphessobrycon tortuguerae: IUQ 2284, (23) (22.4-36.4mm de LE) Costa Rica, Río Muerto, Alajuela S., Upala, 25 abr 1968.

\section{RESULTADOS}

\section{Hyphessobrycon chocoensis sp. n.}

(Figs. 1-4)

Holotipo: IUQ 3035, 37.1mm de LE, macho, Colombia, Nariño, Barbacoas, Quebrada la Tundera afluente del río Quigualpí, $01^{\circ} 39$ $39 \mathrm{~N}-78^{\circ} 0904 \mathrm{~W}, 45 \mathrm{msnm}, 16$ jul 2008. Cols. C. Román-Valencia, C. García-Alzate, R. Ruiz-C, V. Tovar Sr, V. Tovar Jr. \& H. Cortes.

Paratipos: IUQ 2275, (9) $(20.3-37.7 \mathrm{~mm}$ de LE) y IUQ 3036, (1 C\&T) $(36.7 \mathrm{~mm}$ de LE) recolectados con el holotipo; IUQ 2274, (39) (15.9-39.6mm de LE), Colombia, Nariño, Barbacoas, Alto río Yamundé afluente del río Telembí, $01^{\circ} 3939 \mathrm{~N}-78^{\circ} 0904 \mathrm{~W}, 43$

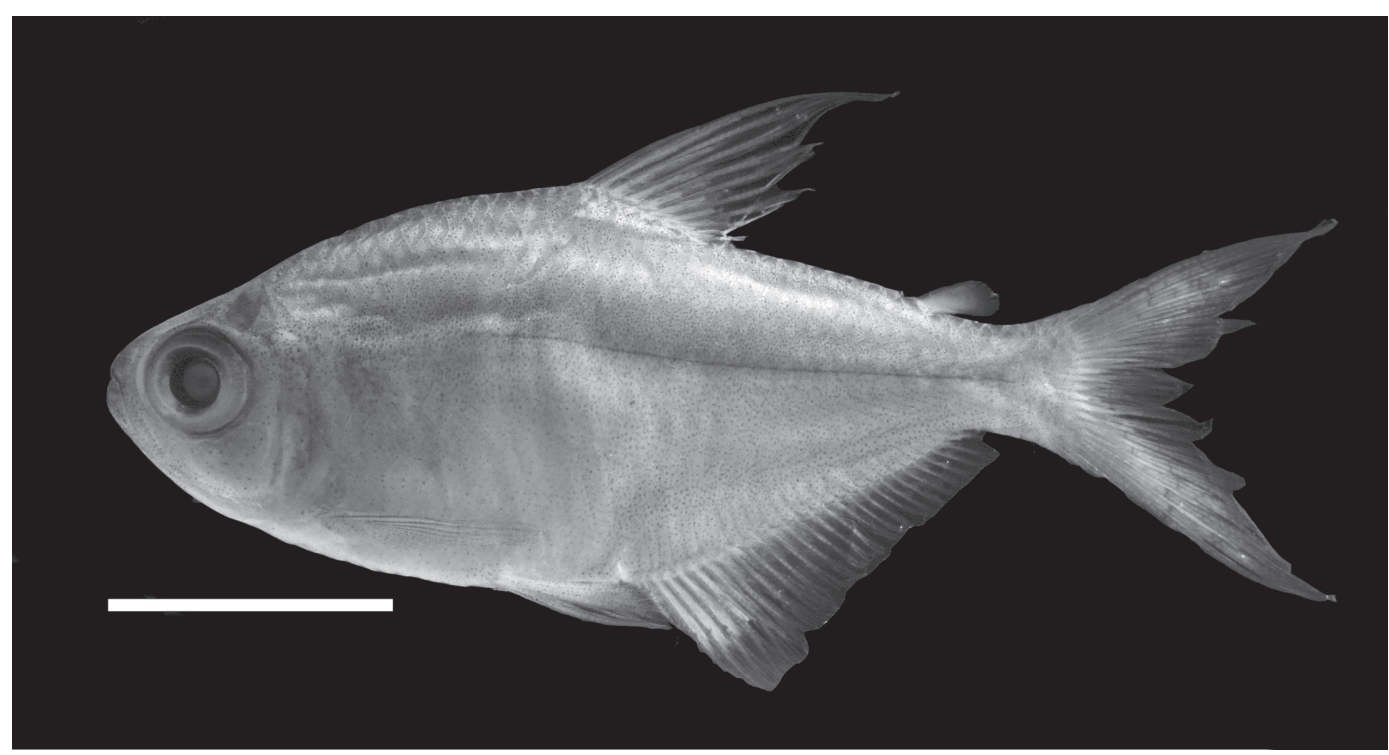

Fig. 1. Hyphessobrycon chocoensis sp. n., IUQ 3035, holotipo, 37.1mm de LE, macho; Quebrada la Tundera, cuenca del río Quigualpí, en Barbacoas, Nariño, Colombia. Escala=1cm.

Fig. 1. Hyphessobrycon chocoensis n. sp., IUQ 3035, holotype, 37.1mm LS, male; Creek la Tundera, Quigualpí river basin, in Barbacoas, Nariño, Colombia. Scale $=1 \mathrm{~cm}$. 
msnm, 16 jul 2008, Cols. C. Román-Valencia, C. García-Alzate, R. Ruiz-C, V. Tovar Sr, V. Tovar Jr. \& H. Cortes; AUM 55075, (3) (17.924.6mm de LE) Colombia, Nariño, Barbacoas, Alto río Yamundé afluente del río Telembí, $01^{\circ} 3939 \mathrm{~N}-78^{\circ} 0904 \mathrm{~W}, 43 \mathrm{msnm}, 16$ jul 2008, Cols. C. Román-Valencia, C. GarcíaAlzate, R. Ruiz-C, V. Tovar Sr, V. Tovar Jr. \& H. Cortes; IUQ 3044, (3 C\&T) (16.4-35.6mm de LE), Colombia, Nariño, Barbacoas, Alto río Yamundé afluente del río Telembí, 01³9 $39 \mathrm{~N}$ - $78^{\circ} 0904$ W, 43 msnm, 16 jul 2008, Cols. C. Román-Valencia, C. García-Alzate, R. Ruiz-C, V. Tovar Sr, V. Tovar Jr. \& H. Cortes.

Diagnosis: Hyphessobrycon chocoensis se distingue de sus congéneres no miembros del grupo "flammeus" por tener una mancha humeral difusa, no tener una mancha oscura en la aleta dorsal o en el pedúnculo caudal. Se distingue de las demás especies del grupo por el número de radios de la aleta dorsal (ii, 8 , i vs. iii, 8 ; ii, 9), por el número de radios ramificados en la aleta anal (25-26 vs. 15-24; excepto $H$. sebastiani con 25-28) y por presentar la mancha humeral difusa (vs. una o dos manchas humerales bien definidas), se diferencia de $H$. tortuguerae por el número de dientes en el maxilar (1-2 vs. 3-10), por el número de escamas predorsales (10-12 vs. 13-15) y un alto número de escamas entre la línea lateral y la aleta anal (6-7 vs. 5). Hyphessobrycon chocoensis se puede separar de todos los otros Hyphessobrycon del grupo flammeus conocidos de las cuencas del Pacifico Colombiano por presentar: un alto número de escamas con poros en la línea lateral (13-31 vs. 7-10); por la longitud hocico-aleta dorsal (41.3-52.3\% LE vs. 53.6-57.5 \% LE); por la profundidad del pedúnculo caudal (7.4-10.5 \% LE vs. 11.2-17.8 $\%$ LE); por la longitud del hocico (16.8-18.0\% LC vs. 18.3-30.7\% LC). Además, se distingue de $H$. columbianus por la longitud aleta dorsalaleta anal (32.7-42.9 \% LE vs. 44.0-46.1\% LE); por la longitud de la mandíbula superior (13.7-16.4 \% LC vs. 18.2-30.7\% LC); por la longitud del hocico (16.8-18.0 \% LC vs. 21.0$30.8 \%$ LC). Del mismo modo se diferencia de $H$. condotensis por tener un alto número de escamas entre la línea lateral y la aleta dorsal (7-8 vs. 6), por una mayor cantidad de radios simples en la aleta anal (iv vs. iii). H. chocoensis se aparta de $H$. sebastiani por el número de escamas en: la parte lateral del cuerpo (31-33 vs. 35-36), entre la línea lateral y la aleta dorsal (7 vs. 8); predorsales (10-11 vs. 12-13).

Descripción: Datos morfométricos del holotipo y paratipos se presentan en el cuadro 1. Cuerpo corto y profundo, la profundidad del cuerpo mayor entre la inserción de las aletas pélvicas y el origen de la aleta dorsal. Perfil dorsal parcialmente cóncavo desde el labio superior hocico hasta el extremo posterior de la espina supraoccipital, y convexo desde este punto hasta el inicio de la aleta dorsal. Base de la aleta dorsal convexa, e inmediatamente después recta hasta la parte anterior de la aleta adiposa y cóncava desde acá hasta la base del lóbulo caudal superior. Perfil ventral de la cabeza convexa desde el labio inferior hasta la inserción de la aleta anal, base de la aleta anal moderadamente convexo y desde allí hasta el lóbulo caudal inferior cóncavo.

Cabeza y hocico cortos, boca terminal; labios blandos y flexibles, hilera externa de dientes del premaxilar no expuesta. Premaxilar con el proceso lateral largo y agudo que se inserta en el etmoides, con dos hileras de dientes (Fig. 2), serie externa con tres (26) ó cuatro* (23) dientes tricúspides, serie interna con cinco (49) dientes pentacúspides que, disminuyen gradualmente de tamaño a medida que se aleja de la sínfisis. Maxilar largo y angosto con dos (49) dientes tricúspides, margen posterior recto y anterior convexo, extremo posterior no alcanza el borde ventral del segundo infraorbital. Dentario con margen ventral convexo, cinco (49) dientes frontales pentacúspides seguido por cinco* (30) ó seis (19) dientes tricúspides más pequeños (Fig. 2).

Escamas cicloideas, línea lateral con 13 (12), $14(15), 31^{*}(22)$ escamas con poros. Serie lateral con $31^{*}(7), 32$ (7), 33 (35) escamas, incluye las escamas con poros. Escamas horizontales entre el origen de la aleta dorsal y 

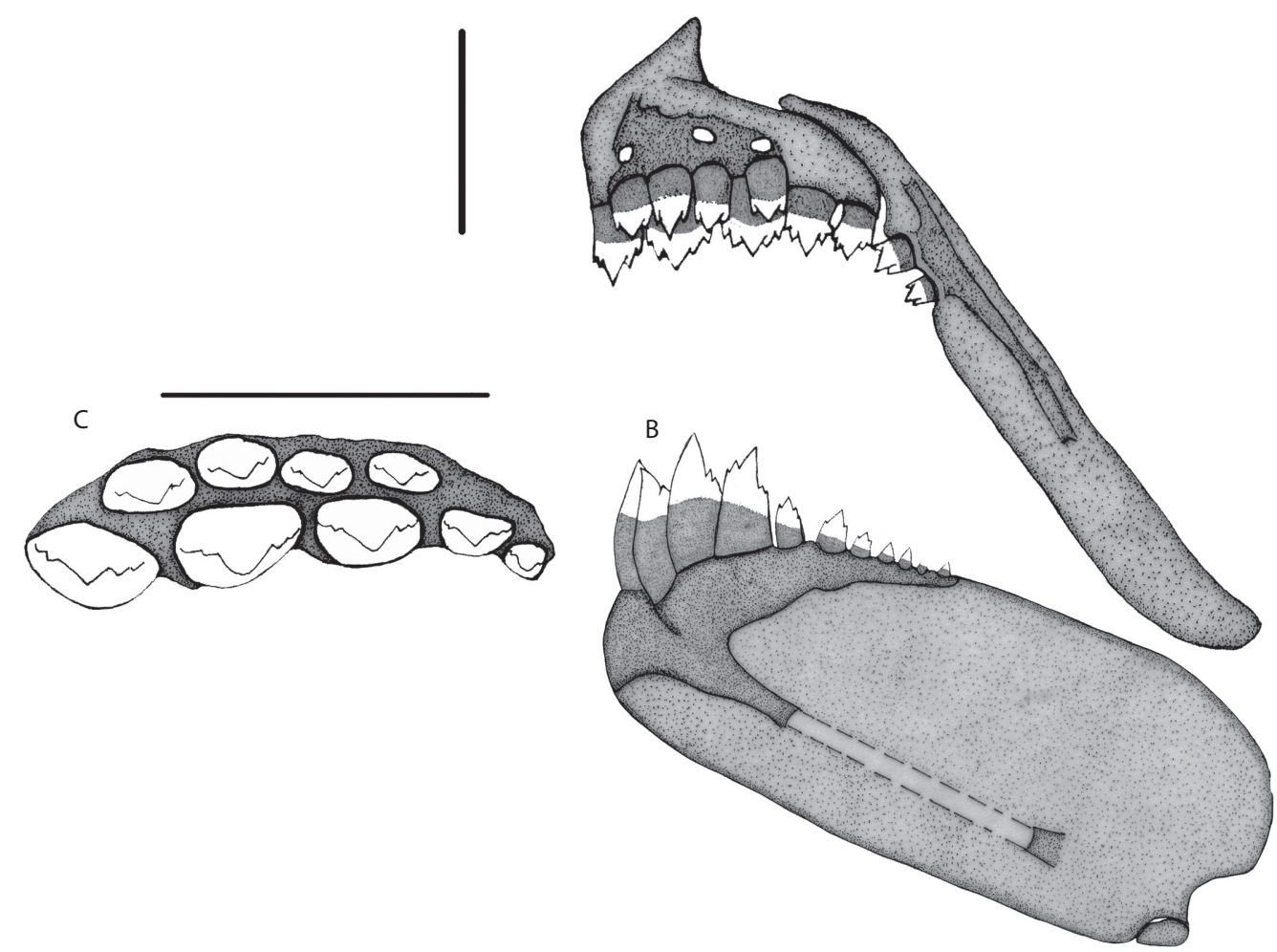

Fig. 2. Hyphessobrycon chocoensis sp. n. IUQ 3036, paratipos. Mandíbulas: superior (A), inferior (B) lado izquierdo, vista ventral del premaxilar (C). Escala $=1 \mathrm{~mm}$.

Fig. 2. Hyphessobrycon chocoensis n. sp. IUQ 3036, paratypes. Jaws: upper (A), lower (B) left side, ventral view of premaxilla (C). Scale bar $=1 \mathrm{~mm}$.

la línea lateral sin incluir las escamas de la serie predorsal situada justo en la parte anterior del primer radio de la aleta dorsal siete* (44), ocho (5). Cinco (49) escamas horizontales entre el origen de la aleta anal y la línea lateral. Cinco (49) escamas horizontales entre el origen de las aletas pélvicas y la línea lateral. Escamas predorsales 10 (30), 11* (19). Cinco* (46) ó siete (3) escamas en una simple hilera en la base anterior de los radios de la aleta anal.

Radios en la aleta dorsal ii, 8, i (49). Radios en la aleta anal iii* (9) o iv (49), 25* (34) o 26 (15). Radios en las aletas pélvicas i, 7 (6), ii, 6* (43). Radios en las aletas pectorales ii, 10*(49). Aleta caudal bifurcada, lóbulos superiores e inferiores puntiagudos y similares en tamaño. Radios principales de la aleta caudal 9+10 (1). Ocho radios procurrentes de la aleta caudal. Número total de vértebras 33.
Ectopterigoide alargado y estrecho se inserta sobre el margen anterior del cuadrado y se extiende por el margen antero ventral del mesopterogoide con una banda de cartílago sobre todo el margen superior. Metapterigoide con una proyección ventral que se contacta con el simplectico y se continúa sobre el cuadrado. Seis huesos infraorbitales presentes, tercer infraorbital más ancho de la serie, no en contacto con el canal laterosensorial del preopérculo, sexto infraorbital más pequeño de la serie, canal sensorial de los infraorbitales sobrepasan el primer infraorbital y se continúa hacia el maxilar. Supraorbital ausente. Etmoides lateral es un hueso largo y cóncavo que se conecta por cartílago al margen lateral del frontal y con una prolongación laminar ósea que se extiende hacia el vómer. Rinosfenoide óseo, con una banda de cartílago sobre el margen anterior 
y posterior. Orbitoesfenoide óseo, grande y alargado, presenta cartílago en la región ventral anterior. Primer arco branquial con 19 braquiespinas, dos en el hipobranquial, nueve en el ceratobraquial y ocho en el epibranquial. Pteriogióforos proximales de los radios de la aleta dorsal insertados entre las espinas neurales nueve y 17; 26 pterigóforos proximales en la aleta anal, los dos iniciales insertados entre las espinas hemales 12 y 13, alcanzan el borde ventral del centrum de la espina hemal 13. Cuatro supraneurales alargados, insertados sobre la cuarta y octava espina neural. Aleta pélvica larga, su extremo posterior alcanza el origen de la aleta anal. Hueso pélvico largo, recto y angosto, se observó cartílago en su extremo anterior y con el proceso isquial largo, curvo y con cartílago.

Dimorfismo sexual: Los machos adultos con un par de ganchos óseos grandes, curvos dorsalmente, ubicados a ambos lados del cuarto radio simple y del primer al cuarto radio ramificado de la aleta anal (Fig. 3).

Color en alcohol: Región dorsal y cabeza marrón oscuro. Fuerte concentración de cromatóforos a lo largo del perfil dorsal, más conspicuo en la cabeza. Margen posterior de las escamas dorsales del margen lateral oscuras por una mayor concentración de pequeños cromatóforos. Con una mancha humeral difusa. Aletas translúcidas, con melanóforos concentrados en la margen posterior. Hocico, labios y maxilar marrón claro, margen ventral del cuerpo color amarillo claro. Membranas interradiales de las aletas pares e impares con cromatóforos pequeños, distribuidos a lo largo de los radios (Fig. 1).

Distribución: Hyphessobrycon chocoensis se conoce de su localidad tipo (Fig. 4).

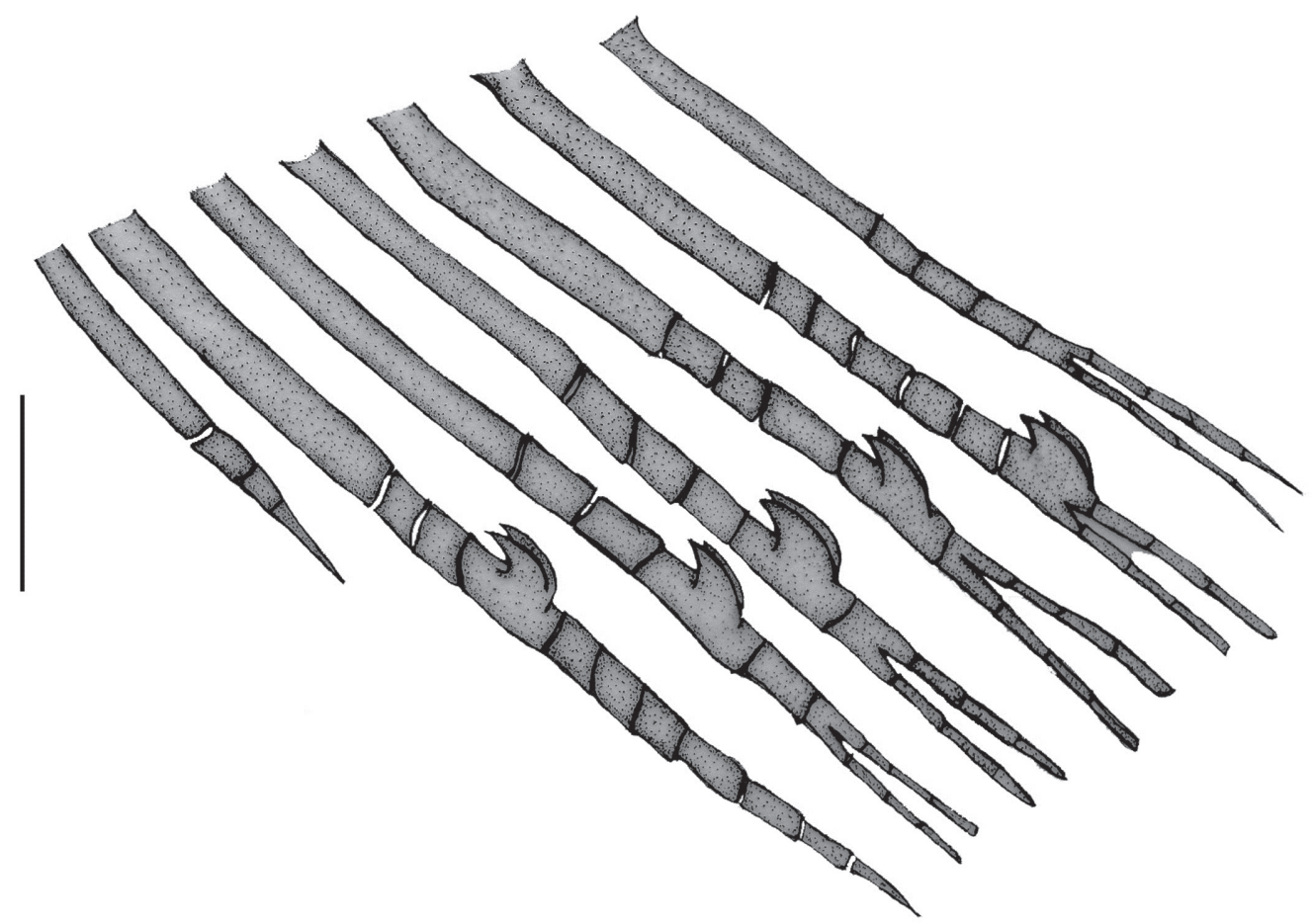

Fig. 3. Hyphessobrycon chocoensis sp. n., IUQ 3036, Paratipos, Vista parcial y lateral de los radios anteriores y espinas de la aleta anal en machos. Lado derecho. Escala $=1 \mathrm{~mm}$.

Fig. 3. Hyphessobrycon chocoensis n. sp. IUQ 3036, Paratypes, Partial view of the anterior rays and spines of the anal fin side and lateral view, in males, the anterior rays and anal fin spines. Right side. Scale bar $=1 \mathrm{~mm}$. 


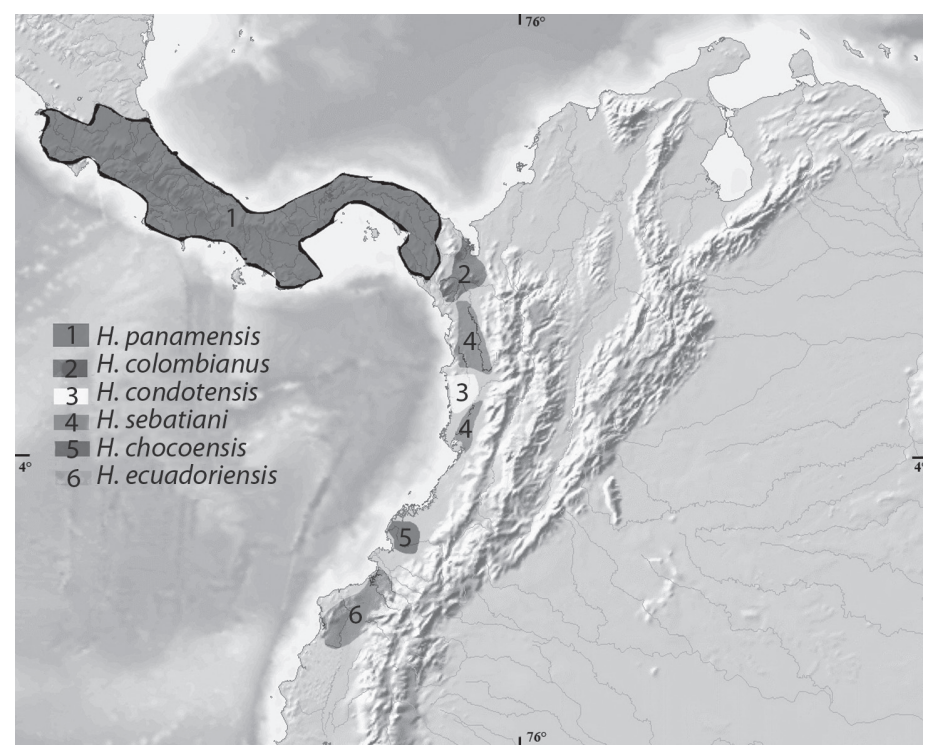

Fig. 4. Mapa de distribución geográfica de las especies de Hyphessobrycon del grupo flammeus presentes en el noroeste de Suramérica y oriente de Centroamérica en Panamá.

Fig. 4. Geographical distribution map of the species of Hyphessobrycon flammeus-group present in northwestern of South America and eastern Central America in Panama.

Etimología: Epíteto específico alusivo al chocó biogeográfico área de distribución de las especies del grupo flammeus en Colombia.

Comentarios: El análisis de componentes principales (ACP) mostró diferencias entre $H$. sebastiani, $H$. panamensis e $H$. chocoensis en el primer componente por la longitud de la aleta anal, la longitud de las aletas pélvicas y la longitud del pedúnculo caudal como las variables más importantes. En el segundo componente la longitud postorbital de la cabeza, la longitud de la mandíbula superior y la longitud de hocico fueron las más importantes, mientras que el componente tres fue mejor explicado por la longitud del maxilar y la longitud de hocico. El primer componente explicó el $81.93 \%$ de la variabilidad total y el segundo componente el $15.67 \%$, entre el primer y tercer componente se explicó el $98.15 \%$ de la variación (Fig. 5, Cuadro 2).

\section{Clave taxonómica de las especies de Hyphessobrycon presentes en la vertiente del Pacífico, Colombia}

1. Manchas humerales presentes; dos radios simples y nueve ramificados en la aleta dorsal; 33 a 36 escamas en la serie

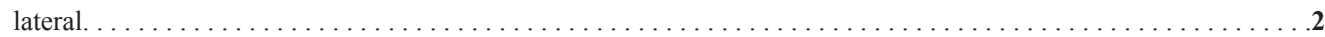

1.1. Manchas humerales ausentes o difusas; tres radios simples y ocho ramificados en la aleta dorsal; 29 a 31 escamas en

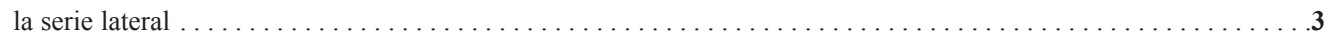

2. Una mancha humeral conspicua; aleta anal con tres a cuatro radios simples; 11 radios ramificados en la aleta pectoral; 31-33 escamas en la serie lateral; 11 escamas con poros en la línea lateral; seis escamas entre línea lateral y la aleta dorsal; 11 escamas predorsales. . . . . . . . condotensis

2.1. Dos manchas humerales conspicuas; aleta anal con cinco radios simples; 12 a 13 radios ramificados en la aleta pectoral; 35 a 37 escamas en la serie lateral; 13 a 18 escamas con poros en la línea lateral; ocho escamas entre línea lateral y la aleta dorsal; 12 a 13 escamas predorsales.

H. sebastiani 
3. 10 a 11 escamas con poros en la línea lateral; longitud hocico-aleta dorsal en la LE 53.6-57.2\%; longitud aleta dorsalaleta anal en la LE 44.0-46.1\%; longitud de la mandíbula superior en la LC 18.2-30.7\%; longitud del hocico en la LC 21.0-30.8\%; dos a tres pares de ganchos óseos en la aleta anal de machos maduros .

H. columbianus

3.1. 13 a 31 escamas con poros en la línea lateral; longitud hocico-aleta dorsal en la LE 41.3-52.3\%; longitud aleta dorsalaleta anal en la LE 32.7-42.9\%; longitud de la mandíbula superior en la LC 13.7-16.4\%; longitud del hocico en la LC 16.8-18.0\%; cuatro a cinco pares de ganchos óseos en la aleta anal de machos maduros ....... H. chocoensis sp. n.

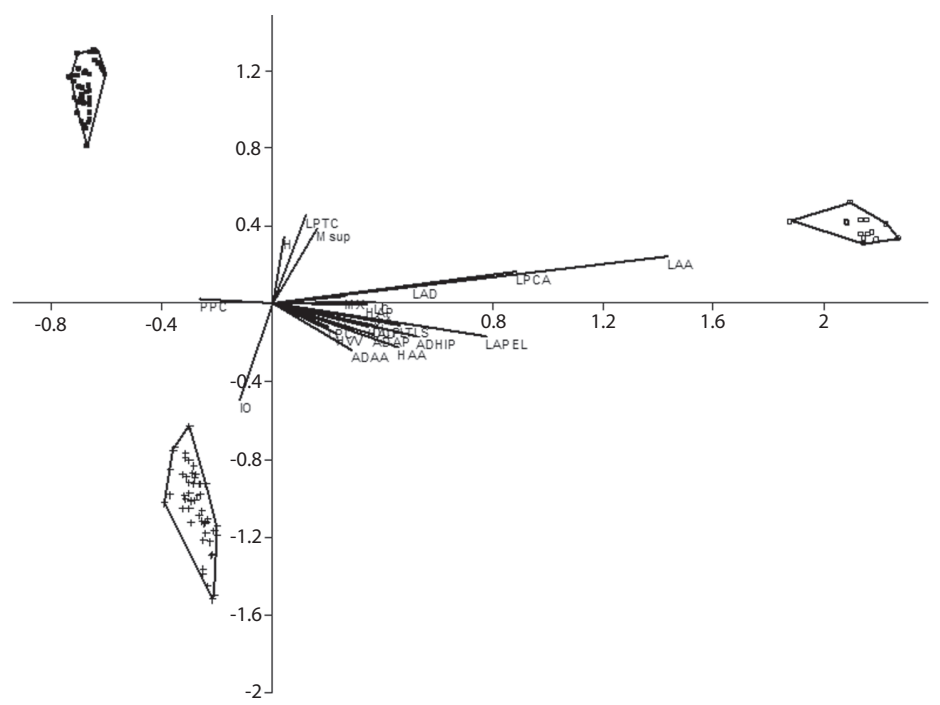

Fig. 5. Análisis de componentes principales de la morfometría de Hyphessobrycon panamesis ( $\square)$, H. sebastiani (+) y H. chocoensis sp. n. (I); componente 1 en el eje horizontal y componente 2 en el eje vertical.

Fig. 5. Principal component analysis of the morphometry of Hyphessobrycon panamesis ( $\square)$, H. sebastiani $(+)$ and $H$. chocoensis n. sp. (I), component 1 in the horizontal axis and component 2 in the vertical axis.

\section{DISCUSION}

Mirande (2010) y Oliveira et al. (2011) en sus análisis con algunas especies del género plantean que Hyphessobrycon es parafilético, sin embargo, sus resultados permiten dilucidar que es posible hallar unidades o grupos naturales dentro del género Hyphessobrycon como lo hemos planteado en sendos trabajos. Weitzman \& Palmer (1997), García-Alzate (2009), García-Alzate et al. (2008a, b, c, 2010 a, b, c, 2011) demuestran que los modelos de coloración presentes en Hyphessobrycon pueden ser útiles como caracteres taxonómicos y en sistemática entre algunas especies de este género y podrían ser la base para evaluar sus relaciones filogenéticas.
Las especies de Norteamérica: Hyphessobrycon compressus (especie tipo del género Hyphessobrycon), y Centroamérica H. tortuguerae, H. savagei, H. milleri y H. panamensis registran tres sinapomorfias: 1- siete dientes en la fila interna del premaxilar, 2- cuadrado con un foramen redondeado en la región ventral y 3- margen ventral del epiótico con dos forámenes pequeños (García-Alzate, 2009). Estas especies están estrechamente relacionadas con las que se distribuyen en el Chocó biogeográfico, caso de $H$. columbianus, $H$. sebastiani, $H$. condotensis, H. ecuadoriensis y H. chocoensis. Esto conlleva a concluir que las especies cisandinas probablemente no sean miembros del género Hyphessobrycon (sensu stricto); en este sentido Weitzman \& Malabarba (1998), S. H. 
CUADRO 2

Vectores propios de los componentes principales 1 a 3 entre Hyphessobrycon sebastiani, H. panamensis y H. chocoensis sp. n. Las abreviaciones (LE, LT, DCP, HAD...) corresponden a las 23 medidas morfométricas del cuadro 1

TABLE 2

Eigenvectors of the principal component 1 to 3 between Hyphessobrycon sebastiani, H. panamensis and H. chocoensis $\mathrm{n}$. sp. The abbreviations (LE, LT, DCP, HAD...) correspond to the 23 morphometric measurements of table 1

\begin{tabular}{lccc} 
& CP1 & CP2 & CP3 \\
LE & 0.2148 & -0.1093 & 0.03562 \\
LT & 0.1923 & -0.109 & -0.02844 \\
DCP & 0.1521 & -0.1082 & 0.03006 \\
HAD & 0.145 & -0.1175 & 0.008788 \\
HAP & 0.1427 & -0.008452 & -0.01911 \\
HVV & 0.09591 & -0.1513 & 0.02292 \\
HAA & 0.191 & -0.2195 & 0.02113 \\
ADHIP & 0.2186 & -0.1596 & 0.0362 \\
ADAA & 0.119 & -0.2277 & -0.02078 \\
ADAP & 0.1533 & -0.1569 & 0.000898 \\
LAD & 0.2127 & 0.07919 & -0.1816 \\
LPC & 0.08417 & -0.1126 & -0.1347 \\
LAPEL & $\mathbf{0 . 3 2 4 2}$ & -0.1652 & -0.0485 \\
LAA & $\mathbf{0 . 5 9 9 3}$ & 0.2308 & -0.0738 \\
PPC & -0.1105 & 0.02077 & -0.1005 \\
LPCA & $\mathbf{0 . 3 7 0 1}$ & 0.1542 & 0.1734 \\
LC & 0.1545 & 0.003471 & 0.002325 \\
H & 0.01744 & $\mathbf{0 . 3 2 5 4}$ & $\mathbf{0 . 7 1 5 3}$ \\
O & 0.09932 & -0.05209 & 0.01655 \\
LPTC & 0.05131 & $\mathbf{0 . 4 3 0 7}$ & -0.5152 \\
MX & 0.1082 & 0.03186 & $\mathbf{0 . 3 2 3 4}$ \\
IO & -0.04945 & -0.4738 & -0.03578 \\
MSUP & 0.06734 & $\mathbf{0 . 3 6 6 5}$ & -0.1077 \\
\hline & & &
\end{tabular}

Weitzman (com. pers.) y García-Alzate et al. (2008b) indicaron que alrededor del 90\% de las especies actualmente asignadas a Hyphessobrycon deben incluirse en otros géneros.

Los peces de la familia Characidae generalmente poseen ganchos en las aletas anal, pélvica, pectoral y más raramente en la dorsal y caudal. El arreglamiento de los ganchos en la aleta anal parecidas a armaduras anales como lo cita Eigenmann (1918) no son muy comunes en Hyphessobrycon. Además, la presencia de ganchos, pero con diferente condición solo están presentes en $H$. compressus, $H$. savagei, $H$. tortuguerae, $H$. panamensis, $H$. ecuadoriensis, H. sebastiani y $H$. chocoensis (Fig. 3, García-Alzate et al. 2010a: Fig. 3); lo que podría sugerir algún tipo de relación entre estas especies.

Dahl (1971) reportó a $H$. panamensis en los ríos San Juan, Atrato y en la desembocadura del río Magdalena. Otros autores (Weitzman \& Palmer 1997, Bussing 1987, Zarske \& Géry 2002, Lima \& Malabarba en Reis et al. 2003) han planteado que $H$. panamensis se distribuye en todas estas áreas. Sin embargo, esta especie corresponde a $H$. sebastiani, e $H$. panamensis se distribuye sólo en Panamá y Costa Rica. Entonces en la actualidad las especies del grupo flammeus transandinas presentan la siguiente distribución: $H$. columbianus en la parte baja del río Atrato, en el río Acandi y sus tributarios, mientras $H$. condotensis en el río Condoto y cuenca baja del río San Juan, $H$. sebastiani en la cuenca media del río San Juan, en la cuenca alta del río Atrato y en sus tributarios, H. chocoensis en el río Quigualpí (cuenca del río Telembi) y finalmente $H$. ecuadoriensis desde el río Mira pasando hasta la cuenca del río Santiago en la costa pacífica de Ecuador.

\section{AGRADECIMIENTOS}

Se recibió financiación de la Universidad del Quindío - Vicerrectoría de Investigaciones (Proyecto 464 y 553). A las siguientes personas por permitir acceso al material bajo su cuidado: Mark Sabaj Pérez (ANSP), Janeth Muñoz Saba (ICMNH), Karsten Hartel (MCZ), Gustavo Casas Andreu (IBUAM-P), Francisco Provenzano (MBUCV) y Carlos Alberto Lucena (MCP). John Fong (CAS) y James Maclaine (BMNH) por el envío de las fotografías del material tipo. La familia Tovar (María, Jhon, Víctor padre e hijo) por su generosa hospitalidad y ayuda en Barbacoas, Nariño. Raquel Ruiz-C (IUQ) asistió en el trabajo de campo y Juan D. García en laboratorio. 


\section{RESUMEN}

El género Hyphessobrycon, se incluye dentro de la subfamilia Tetragonopterinae. Las especies generalmente son pequeñas, no sobrepasan los $70 \mathrm{~mm}$ de longitud estándar, son importantes económicamente como peces ornamentales, con 128 especies validas que se distribuyen desde el sur de México hasta Río de La Plata en Argentina. Se describe una nueva especie de Hyphessobrycon (Characiformes: Characidae) de la cuenca del río Barbacoas, vertiente sur del Pacífico, Colombia, Hyphessobrycon chocoensis de la cuenca del río Telembí, vertiente del Pacífico Colombiano. Las recolectas de los peces fueron realizadas con redes de arrastres y se hicieron en un solo biotopo, zona litoral de remanso y a favor de la corriente. Las medidas de los ejemplares se tomaron punto a punto con un calibrador digital Las observaciones de estructuras óseas y cartílagos se hicieron sobre ejemplares clareados y teñidos (C\&T). Las relaciones morfométricas entre especies fueron exploradas empleando 21 variables mediante un análisis de componentes principales (ACP). La nueva especie de Hyphessobrycon, H. chocoensis se distingue de sus congéneres no miembros del grupo "flammeus" por tener una mancha humeral difusa, no tener una mancha oscura en la aleta dorsal o en el pedúnculo caudal. Se distingue de las demás especies del grupo por el número de radios de la aleta dorsal (ii, 8 , i), por el número de radios ramificados en la aleta anal (25-26) y por presentar la mancha humeral difusa, se diferencia de $H$. tortuguerae por el número de dientes en el maxilar (1-2), por el número de escamas predorsales (10-12) y un alto número de escamas entre la línea lateral y la aleta anal (6-7). Hyphessobrycon chocoensis se puede separar de todos los otros Hyphessobrycon conocidos de las cuencas del Pacifico Colombiano por presentar: un alto número de escamas con poros en la línea lateral, por la longitud hocico-aleta dorsal, por la profundidad del pedúnculo caudal y por el diámetro del ojo. Además, se distingue de $H$. columbianus por la longitud aleta dorsalaleta anal, por la longitud de la mandíbula superior, por la longitud del hocico. Del mismo modo se diferencia de $H$. condotensis por tener un alto número de escamas entre la línea lateral y la aleta dorsal, por una mayor cantidad de radios simples en la aleta anal. $H$. chocoensis se aparta de $H$. sebastiani por el número de escamas en la parte: lateral del cuerpo y entre la línea lateral y la aleta dorsal. Se incluye una clave taxonómica práctica de las especies presentes en la vertiente del Pacifico de Colombia.

Palabras clave. nuevo taxón, nueva especie, pez neotropical, Chocó biogeográfico, sardina.

\section{REFERENCIAS}

Bussing, W.A. 1987. Peces de las aguas continentales de Costa Rica. Universidad de Costa Rica, San José, Costa Rica.

Dahl, G. 1971. Los Peces del Norte de Colombia. Inderena, Bogotá, Colombia.
Eigenmann, C.H. 1918. The American Characidae. Part 2. Mem. Mus. Comp. Zoo. 43: 103-208.

Eschmeyer, W.N. \& R. Fricke. 2011. Catalog of Fishes electronic version. California Academy of Sciences, California, EEUU. (Consultado: 24 agosto 2011, http://www.calacademy.org/research/ichthyology/ catalog/fishcatsearch.html).

Fink, W.L. \& S.H. Weitzman. 1974. The so-called Cheirodontin fishes of Central America with descriptions of two new species (Pisces: Characidae). Smith. Cont. Zoo. 172: 1-45.

García-Alzate, C.A. 2009. Análisis filogenético y biogeográfico de las especies de Hyphessobrycon (Characiformes: Characidae) grupo heterorhabdus. Tesis de Doctorado, Universidad Central de Venezuela, Caracas, Venezuela.

García-Alzate, C.A. \& C. Román-Valencia. 2008. Hyphessobrycon ocasoensis sp.n. (Teleostei, Characidae) una nueva especie para el Alto Cauca, Colombia. Anim. Biol. Conserv. 31: 11-23.

García-Alzate, C.A., C. Román-Valencia \& D.C. Taphorn. 2008a. Hyphessobrycon oritoensis (Characiformes: Characidae), a new species from the Putumayo River drainage, Colombian Amazon. Zootaxa 1813: 42-50.

García-Alzate, C.A., C. Román-Valencia \& D.C. Taphorn. 2008b. Taxonomic revision of the Hyphessobrycon heterorhabdus-group (Teleostei: Characidae) from Venezuela, with description of two new species. Vert. Zool. 58: 139-157.

García-Alzate, C.A., C. Román-Valencia \& D.C. Taphorn. 2008c. Two new species of the genus Hyphessobrycon (Pisces: Characiformes: Characidae) from Putumayo River, with keys to the Colombian Hyphessobrycon heterorhabdus-group species. Brenesia 40: 33-46.

García-Alzate, C.A., C. Román-Valencia \& D.C. Taphorn. 2010a. A new species of Hyphessobrycon (Teleostei: Characiformes: Characidae) from the San Juan River drainage, Pacific versant of Colombia. Zootaxa 2349: 55-64.

García-Alzate, C.A., C. Román-Valencia \& M. González. 2010b. Análisis morfogeométrico de las especies de Hyphesssobrycon grupo heterohabdus de Venezuela. Rev. Biol. Trop. 58: 801-811.

García-Alzate, C.A., C. Román-Valencia \& S. Prada-Pedreros. 2010c. Tres nuevas especies de Hyphessobrycon grupo heterorhabdus (Teleostei: Characiformes: Characidae), con clave para especies de la cuenca del río Orinoco. Caldasia 32: 415-433.

García-Alzate, C.A., R. Ruiz-C., C. Román-Valencia, M. González \& D. Lopera. 2011. Morfología de las especies de Hyphessobrycon (Characiformes: Characidae), grupo heterorhabdus, en Colombia. Rev. Biol. Trop. 59: 709-725. 
Géry, J. 1977. Characoids of the World. THF, Neptune City, Nueva Jersey, EEUU.

Hammer, Ø., D.A.T. Harper \& P.D. Ryan. 2008. PAST: Paleontological Statistics, ver. 1.81. Paleontological Museum, University of Oslo, Noruega. (también disponible en línea: http://folk.uio.no/ohammer/past/ index.html).

Mirande, M. 2010. Phylogeny of the family Characidae (Teleostei: Characiformes): from characters to taxonomy. Neot. Ichth. 8: 385-568.

Oliveira, C., G.S. Avelino, K.T. Abe, T.C. Mariguela, R.C. Benine, G. Orti, R.P. Vari, R.M. Correa. 2011. Phylogenetic relationships within the speciose family Characidae (Teleostei: Ostariophysi: Characiformes) based on multilocus analysis and extensive ingroup sampling. BMC Evol. Biol. 11: 275.

Reis, R.E., S.O. Kullander \& C.J.Jr. Ferraris. 2003. Check list of the freshwater fishes of South and Central America. CLOFFSCA. i-xi + 1-729.

Sabaj-Perez, N.H. 2010. Standard symbolic codes institutions resource collections in Herpetology and Ichthyology: an on line reference, version 1.5. American Society Ichthyologist and Herpetologist, Washington, D.C., EEUU. (Consultado: 04 octubre 2012, http.// www.asih.org/).

Song, J. \& L. Parenti. 1995. Clearing and staining whole fish specimens for simultaneous demonstration of bone, cartilage and nerves. Copeia 1995: 114-118.
Taylor, W.R. \& G.C. Van Dyke. 1985. Revised procedures for staining and clearing small fishes and other vertebrates for bone and cartilage study. Cybium 9: 107-119.

Vari, R.P. 1995. The neotropical fish family Ctenoluciidae (Teleostei: Ostariophysi: Characiformes) supra and intrafamilial phylogenetic relationships, with a revisionary study. Smith. Cont. Zoo. 564: 1-96.

Weitzman, S.H. 1962. The osteology of Brycon meeki, a generalized characid fish, with an osteological definition of the family. Stan. Ichth. Bull. 8: 3-77.

Weitzman, S.H. \& L. Palmer. 1997. A new species of Hyphessobrycon (Teleostei: Characidae) from the Neblina region of Venezuela and Brazil, with comments on the putative "rosy tetra clade". Ichth. Explor. Fresh. 7: 209-242.

Weitzman, S.H. \& L.R. Malabarba. 1998. Perspectives about the Phylogeny and Classification of the Characidae (Teleostei: Characiformes), p. 161-169. In L.R. Malabarba, R.E. Reis, R.P. Vari, Z.M.S. Lucena \& C.A.S. Lucena (eds.). Phylogeny and Classification of Neotropical Fishes. EDIPUCRS, Porto Alegre, Brasil.

Zarske, A. \& J. Géry. 2002. Der Blaurote KolumbienSalmler Hyphessobrycon columbianus n. sp. - ein neuer Salmler (Teleostei; Characiformes, Characidae) aus dem kolumbianischen Darien. Das Aquarium 391: $22-30$ 